\title{
Bulge fatigue testing of freestanding and supported gold films
}

\author{
Benoit Merle $^{a)}$ and Mathias Göken \\ Department of Materials Science and Engineering, Friedrich-Alexander-University Erlangen-Nürnberg (FAU), \\ Institute I, Martensstr. 5, D-91058 Erlangen, Germany
}

(Received 27 September 2013; accepted 25 November 2013)

\begin{abstract}
The bulge test was used to investigate the fatigue properties of gold thin films with a thickness between 100 and $300 \mathrm{~nm}$. The membranes were pressurized at a rate of $0.2 \mathrm{~Hz}$ up to $10^{5}$ times, during which their stress and strain states were continuously recorded. Gold films on a silicon nitride substrate were cyclically loaded into tension and compression. Due to the presence of the substrate, no membrane failure was observed, but the residual stress shifted from an initially tensile state to an increasingly compressive one. Typical fatigue damage mechanisms consisting of extrusions were found in some large grains. Freestanding films were cyclically loaded in pure tension until failure occurred. The data acquired during the fatigue tests show a strong ratcheting of the films, which is indicative of cyclic plastic creep. Microstructural investigations clearly show grain boundary sliding in very thin films with columnar grains extending through the thickness.
\end{abstract}

\section{INTRODUCTION}

Thin films have long been popular research objects for studying the scale dependence of the mechanical properties of materials. The scientific interest has recently grown to a new dimension, as thin films are becoming more and more pivotal in a large number of consumer goods, prompting a strong industrial demand for assessing their reliability.

Most of the research on the fatigue properties of thin films has so far been performed on samples attached to a substrate, with the main focus on copper films and to a lesser extent on gold and silver films. The same general trends have in fact been found for these materials. Thick films usually display similar extrusions and voids at the free surface as known from macroscopic samples. ${ }^{1,2}$ Transmission electron microscopy observations, for instance by Zhang et al. ${ }^{3}$ on $3 \mu \mathrm{m}$ copper films, hint at a similar mechanism of formation of walls and cells dislocation structures. Such similitudes disappear for thinner copper films. In the transition regime corresponding to a thickness of $200 \mathrm{~nm}-1 \mu \mathrm{m}$ in copper, the dislocations are more randomly organized $^{3}$ and extrusions are more rarely found on the surface. ${ }^{1}$ Last, for copper films below $200 \mathrm{~nm}$, damage formation is mostly localized at interfaces such as grain boundaries or twin boundaries. ${ }^{3,4}$ The explanation that has been proposed for explaining the transition in cyclic deformation mechanisms is that the dimensional and microstructural constraints become too strong to allow the formation of organized dislocation structures. ${ }^{5}$ The typical

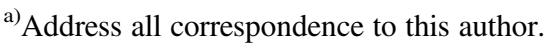

e-mail: benoit.merle@fau.de

DOI: $10.1557 /$ jmr.2013.373
}

formation of extrusions as described by Mughrabi ${ }^{6,7}$ for macroscopic samples is indeed based on the formation of persistent slip bands, which in bulk copper have a characteristic wall-spacing of $1.4 \mu \mathrm{m} .{ }^{8}$ Since the yield strength of thinner films is also higher, these might not be able to deform plastically until a high stress is reached, which is larger than the stress needed for cracking at grain boundaries. ${ }^{4}$ This could explain why grain boundary cracking dominates the fatigue life, while some extrusions can still form if testing is continued after cracking has occurred. ${ }^{9}$ A general observation is that very thin films tested with the same stress amplitude exhibit a longer fatigue life than thicker ones, ${ }^{2}$ whichever definition is adopted for fatigue life. ${ }^{9,10}$ While the general picture of the size effect is clear, there are certain factors that can influence its magnitude or the thickness range for the transition. A good adhesion of the film to the substrate can for instance postpone the local debonding that is needed for the slip bands to develop and thus extend the fatigue life of the sample and possibly change the failure mode to grain boundary cracking. ${ }^{9}$ Another interesting finding from Lu et al. ${ }^{11}$ is that as-deposited copper films with an unstable microstructure are not very fatigue resistant. Such films are indeed subject to local abnormal grain growth during testing, which leads to the creation of weak spots (Hall-Petch effect), where the deformation is immediately localized and extrusions can grow.

Freestanding films with submicrometer thickness have yet hardly been investigated, ${ }^{12-14}$ largely due to the difficult sample handling. As a consequence, their fatigue behavior remains largely unknown. In recent years, the bulge test has emerged as an appropriate technique for investigating the monotonic mechanical properties of very thin freestanding films. ${ }^{15}$ It therefore makes sense to also apply it to the study of their fatigue properties. 


\section{BULGE TESTING}

The self-built experimental setup that has been used for this study relies on gaseous nitrogen for pressurizing a membrane. It can be operated in two distinct modes: ex situ, the deflection of the membrane is continuously measured by a laser autofocus displacement sensor and in situ, the whole setup is inserted into a Dimension 3100 atomic force microscope (AFM; Bruker, Santa Barbara, $\mathrm{CA}$ ), which enables imaging the surface of the sample under load. The experimental setup has been described in more detail elsewhere ${ }^{16}$ and has also been used to measure the fracture toughness of very thin films ${ }^{17}$ in a similar way as pioneered by previous authors. ${ }^{18,19}$

As for the present study, cyclic testing is achieved by repeating a triangular pressure pattern a given number of times. The use of a fast settling exhaust valve allows an operating frequency of $0.2 \mathrm{~Hz}$, which is twice as high as that found for a bulge tester with comparable gas pressure actuation. ${ }^{20}$ Higher frequencies are difficult to achieve with the current experimental setup, due to the limited permitted gas flow. This is the reason why the setup is preferentially used for low cycle rather than high cycle and ultrahigh cycle fatigue testing.

The tested samples have a $4 \times 1 \mathrm{~mm}$ rectangular geometry and a thickness between 100 and $400 \mathrm{~nm}$, which justifies using the analytical model of a long rectangular membrane developed by Vlassak et al. ${ }^{21,22}$ This model enables calculating the lateral stress $\sigma_{x x}$ and strain $\varepsilon_{x x}$ of the film from the pressure-deflection data recorded during the experiment:

$$
\begin{gathered}
\sigma_{x x}=\frac{p a^{2}}{2 h t}, \\
\varepsilon_{x x}=\frac{2 h^{2}}{3 a^{2}}+\varepsilon_{0},
\end{gathered}
$$

where $a$ is the half-width of the membrane, $t$ its thickness, $p$ the applied gas pressure, and $h$ the deflection in the center of the membrane, as indicated in Fig. 1(a). In case the film consists of a bilayer and the mechanical properties of one layer are known, it can easily be shown that the stress experienced by the second layer $\sigma_{x x, 2}$ is given by:

$$
\sigma_{x x, 2}=\frac{t}{t_{2}} \sigma_{x x}-\frac{t_{1}}{t_{2}} \sigma_{x x, 1}
$$

i.e.,

$$
\sigma_{x x, 2}=\frac{p a^{2}}{2 h t_{2}}-\frac{t_{1}}{t_{2}}\left[\frac{E_{\mathrm{Y} 1}}{1-v_{1}^{2}} \cdot \frac{2 h^{2}}{3 a^{2}}+\sigma_{0,1}\right],
$$

where $E_{\mathrm{Y} 1}$ is the known Young's modulus, $v_{1}$ the Poisson's ratio, and $\sigma_{0,1}$ the residual stress of the underlying layer of the sample, see Fig. 1(b). In addition to the implementation of these equations, the script used for the evaluation of the stress-strain data implements a correction for the machine compliance and for an initial

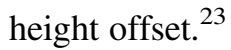

\section{SAMPLE PREPARATION}

The samples consisting of a $1 \times 4 \mathrm{~mm}$ rectangular membrane held by a silicon frame were fabricated by micromachining. In a first step, a silicon wafer was coated on both sides with nonstoichiometric "low-stress" LPCVD (low pressure chemical vapor deposition) $\mathrm{SiN}_{x}$. Next, rectangular openings were then patterned on the backside by means of photolithography, and the silicon was finally etched away from this area with potassium hydroxide. These first two steps were outsourced to Silson Ltd. (Northampton, U.K.). The resulting freestanding $\mathrm{SiN}_{x}$ film was either directly tested in the bulge test or used as a substrate for the deposition of gold. The latter was performed using a PVD MED-020 (Balzers Oerlikon, Liechtenstein) evaporation device. A thin chromium adhesion layer $(<10 \mathrm{~nm})$ and a much thicker gold film $(>100 \mathrm{~nm})$ were successively deposited without interrupting the vacuum. The bilayer gold- $\mathrm{SiN}_{x}$ membranes were tested from this condition, while the freestanding gold films required further processing. In this case, both $\mathrm{SiN}_{x}$ and chromium were removed from the backside of the membrane by hot phosphoric acid etching. This resulted in

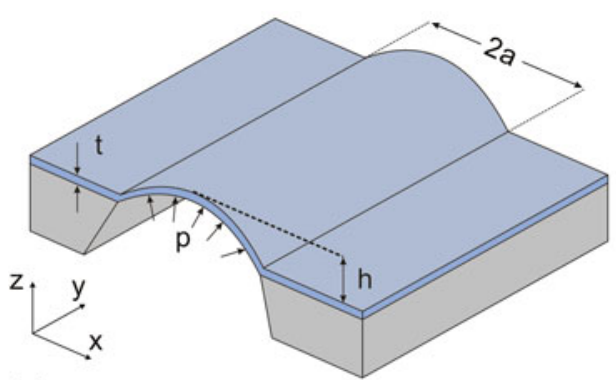

(a)

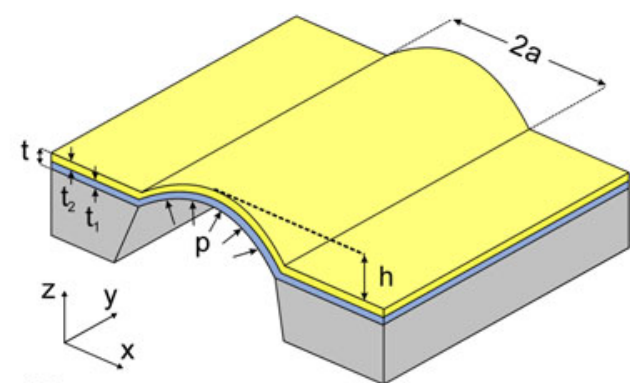

(b)

FIG. 1. Schematic of the central section of a long rectangular membrane during bulge testing: (a) single layer and (b) bilayer membrane. 
the gold film being totally freestanding in the rectangular section and firmly bonded to the frame anywhere else. As already well-documented in the literature, the deposition process used for the gold films resulted in a strong (111) fiber texture ${ }^{24}$ and a predominantly columnar microstructure. $^{25}$

\section{EXPERIMENTAL RESULTS}

\section{A. Validation of the bulge fatigue test with $\operatorname{SiN}_{x}$ membranes}

To validate the new bulge fatigue testing method, measurements were carried out on a plain $\mathrm{SiN}_{x}$ membrane. Amorphous LPCVD-SiN ${ }_{x}$ is indeed known from the literature to have an almost infinite fatigue life, ${ }^{26,27}$ thus making it a perfect material for testing the long-term stability of the experimental setup. In detail, a 100-nm-thin $\mathrm{SiN}_{x}$ film was tested at $0.2 \mathrm{~Hz}$ with a maximum pressure of $50 \mathrm{kPa}$. The membrane withstood $10^{5}$ cycles-corresponding to 6 days-without failing. The plane-strain modulus and residual stress calculated for each cycle are shown in Fig. 2(a). The periodic scattering visible in the residual stress data of Fig. 2(a) results from short interruptions of the test after every 500th cycle for pressure calibration purposes. The plane-strain modulus and the residual stress level remain absolutely constant throughout the whole test
Further interesting information is available from the comparison of the stress-strain diagrams calculated for every 10,000th cycle, as shown in Fig. 2(b). First of all, the loading and unloading segments are linear and overlap perfectly, confirming that the deformation of $\mathrm{SiN}_{x}$ is indeed purely elastic. The lack of hysteresis more importantly proves that no significant machine effects are occurring. Secondly, the diagrams of all cycles overlap extremely well. This is a confirmation that LPCVD $\mathrm{SiN}_{x}$ is not affected by fatigue or by cracking, which would have resulted in a reduction in stiffness and a modification of the slope of the segments. More importantly, it shows that the fatigue test device is very stable over long periods of time.

\section{B. Thin gold films bonded to a $\operatorname{SiN}_{x}$ substrate}

\section{Bulge fatigue testing}

Similar cyclic tests were performed on gold films bonded to a $\mathrm{SiN}_{x}$ substrate. All in all, about ten gold samples with a thickness between 100 and $300 \mathrm{~nm}$ were tested on a 100 -nm-thick $\mathrm{SiN}_{x}$ substrate. Since the results are very similar, only a representative test on a 100-nm-thick gold film will be presented here. This sample was pressurized up to $100 \mathrm{kPa}$ at a frequency of $0.2 \mathrm{~Hz}$. The reason why the maximum pressure was chosen higher than that of the plain $\mathrm{SiN}_{x}$ films is that the total thickness was larger. The recorded experimental data was evaluated by Eq. (4), which
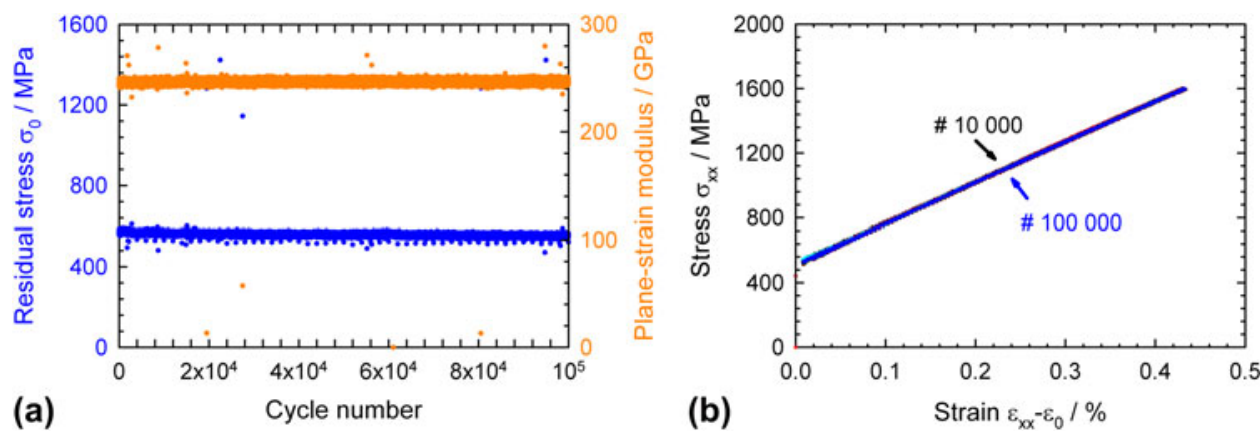

FIG. 2. Quantitative evaluation of the fatigue test of a $100 \mathrm{~nm} \mathrm{SiN}_{x}$ film at $0.2 \mathrm{~Hz}$ and $50 \mathrm{kPa}$ (a) plane-stress modulus and residual stress evolution; (b) stress-strain loops for every 10,000th cycle (the first cycle is skipped as it corresponds to the initial monotonic-like deformation).
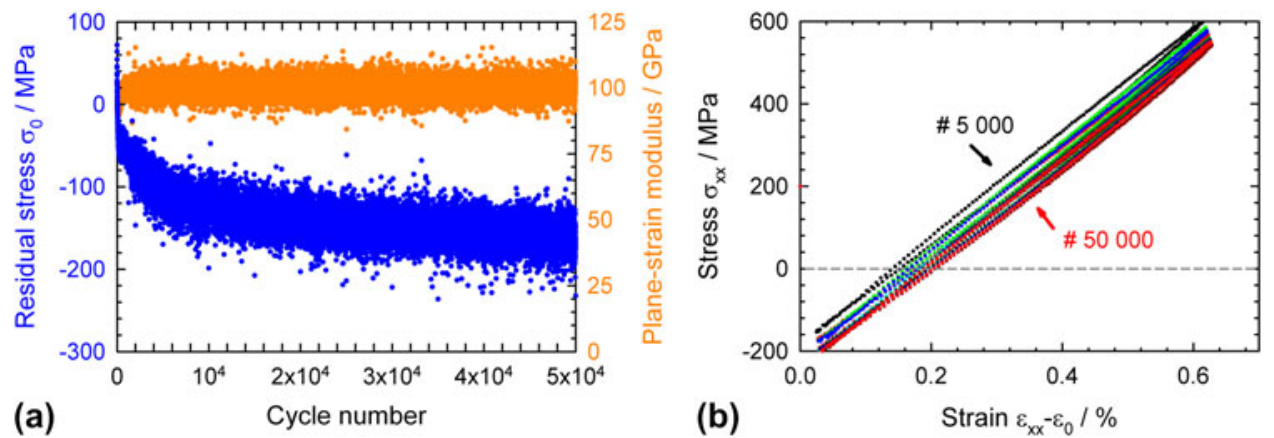

FIG. 3. Quantitative evaluation of the fatigue test for a $100 \mathrm{~nm}$ gold film on $\mathrm{SiN}_{x}$ at $0.2 \mathrm{~Hz}$ and $100 \mathrm{kPa}$ : (a) plane-stress modulus and residual stress evolution and (b) stress-strain loops for every 5000th cycle. 
allowed extracting the contribution of the gold film under the verified assumption that the mechanical properties of the $\mathrm{SiN}_{x}$ substrate remain constant throughout the test. The most important results of the quantitative evaluation are reported in Fig. 3. First of all, it should be noted that the membrane did not fail during the whole 50,000 cycles of testing. This is a general behavior: other samples have even been tested up to 200,000 cycles without failure. The obvious reason is that the $\mathrm{SiN}_{x}$ substrate has an almost infinite fatigue life and can bear the full load on its own. As shown in Fig. 3(a), the scattering in the data appears to be quite large, which is a consequence of the extrapolation of the contribution of the gold layer from the deformation of the overall composite membrane. It can however clearly be seen that the planestrain modulus remains constant throughout the test. The measured value of $100 \mathrm{GPa}$ is higher than the data found in the literature for macroscopic polycrystalline gold samples (93 GPa according to Ref. 28). However, this is consistent with the fact that the sample has a strong (111) texture component: according to the equations provided in Ref. 29 , a sample with a pure (111) texture would have a plane-strain modulus as high as $121 \mathrm{GPa}$ (elastic constants from Ref. 30). Anyway, the stability of the measured plane-strain modulus throughout testing indicates the lack of large cracks and delamination from the substrate. The residual stress shifts in contrast to larger and larger compressive values, although the initial state of the as-deposited sample was tensile $(+100 \mathrm{MPa})$. This evolution is very fast during the first 3000 cycles and continues further, albeit at a decreasing rate. This shift toward more compressive residual stresses can be interpreted as the result of an increased elongation of the film due to successive plastic deformation. This is confirmed by

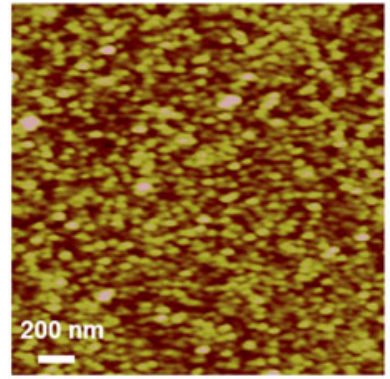

(a)

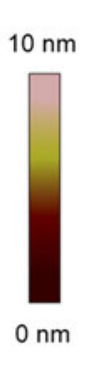

(b)

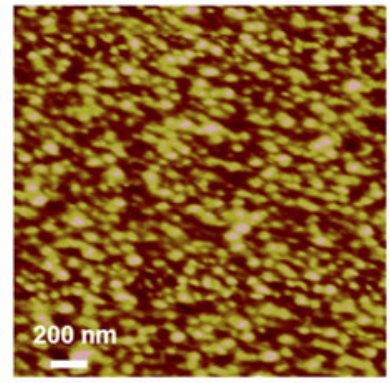

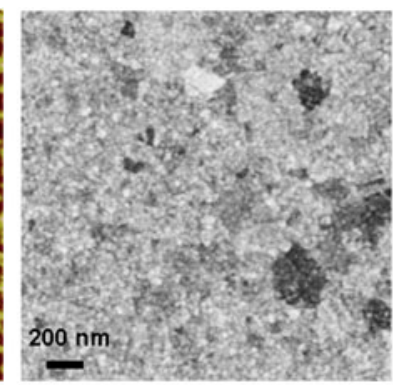

(c)

FIG. 4. Topography of a 120-nm-thick gold layer on $\mathrm{SiN}_{x}$ investigated by AFM: (a) before fatigue testing, (b) after $290,000 \mathrm{cycles}$ at $\Delta p=50 \mathrm{kPa}$, and (c) backscattered electron image taken before testing, showing the underlying microstructure.

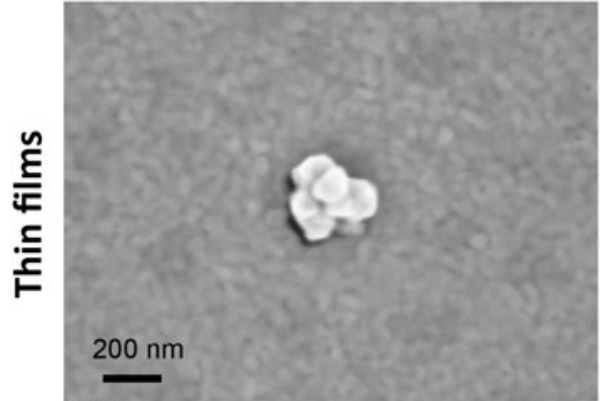

(a)

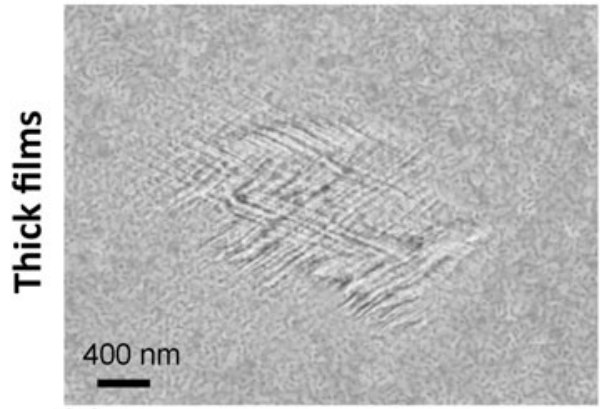

(c)

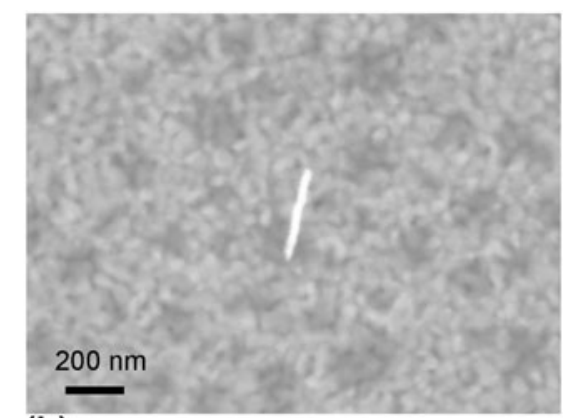

(b)

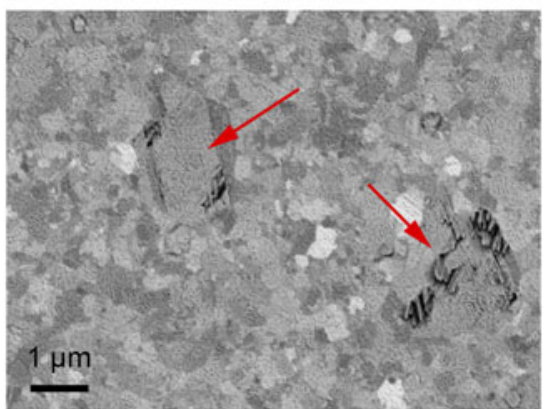

(d)

FIG. 5. Localized damage formation during fatigue testing of supported gold films with different thicknesses. (a and b) Hillock and whisker formation in a $100 \mathrm{~nm}$ gold film and (c and d) extrusions in very large grains of a 300-nm-thick gold film. 
the observation of the hysteresis loops shown in Fig. 3(b), showing a progressive shift toward higher strains. Interestingly, both strain increment and plastic strain amplitude continuously decrease with increasing number of cycles. These combined observations of cyclic creep and cyclic hardening of the film are usually referred to as ratcheting. Another interesting observation from Fig. 3(b) is that supported gold films are subjected to a stress alternating between tension and compression, unlike the freestanding films that can only be loaded in tension. The reason is that, when loaded beyond its yield strength, gold flows plastically, while the brittle $\mathrm{SiN}_{x}$ substrate continues to deform purely elastically. When the load is removed, the $\mathrm{SiN}_{x}$ film contracts and pulls the strongly bonded gold layer into compression. ${ }^{31,32}$

\section{Microscopic observations}

The gold films on $\mathrm{SiN}_{x}$ substrates were investigated in the AFM and the scanning electron microscope (SEM) before and after testing. AFM observations revealed that the fatigue tests amounted to a slight uniform roughening of the gold surface. It should be pointed out that the topography recorded by the AFM does not necessarily reflect the underlying microstructure of the sample. The mean grain size is usually larger than the structures visible on the surface, as evidenced by comparing Figs. 4(a) and 4(c). The hillocks seen at the surface originate from the early condensation islands created during film deposition. The roughening observed during the fatigue test therefore does not correspond to grain growth. This effect is anyway very limited in magnitude: in the representative case of the film shown in Figs. 4(a) and 4(b), the roughness $R_{a}$ increased from 1.1 to $1.5 \mathrm{~nm}$ only.

In addition to this uniform roughening, localized damage formation was observed on several samples tested at a range of $100 \mathrm{kPa}$. Typical micrographs are shown in Fig. 5. Very thin films of about $100 \mathrm{~nm}$ thickness show the growth of hillocks [Fig. 5(a)] and whiskers [Fig. 5(b)] from the surface. Such a behavior is frequently encountered for stressed aluminum and lead films, most of the time- but not always-at elevated temperature. ${ }^{33-35} \mathrm{It}$ is obviously caused by the compressive stress reached in the film upon unloading of the pressure, which induces the material to flow out of plane. Thicker films of about $300 \mathrm{~nm}$ thickness show extrusions forming inside very large grains [Figs. 5(c) and 5(d)]. Their occurrence in the largest grains is not a coincidence, as plasticity is facilitated there by the Hall-Petch effect. A similar localization in large grains has been observed by Lu et al. ${ }^{11}$ in copper films tested on a polymer substrate.

\section{Freestanding gold films}

\section{Bulge fatigue testing}

The freestanding gold films showed a more pronounced fatigue behavior than the films on a substrate. This is well illustrated by the measurement performed on a 230 -nm-thick
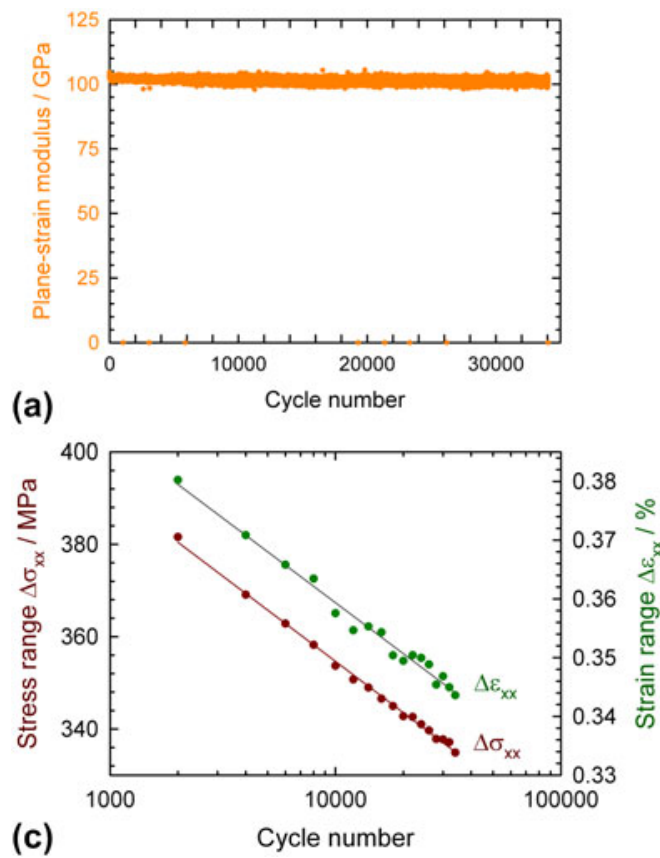
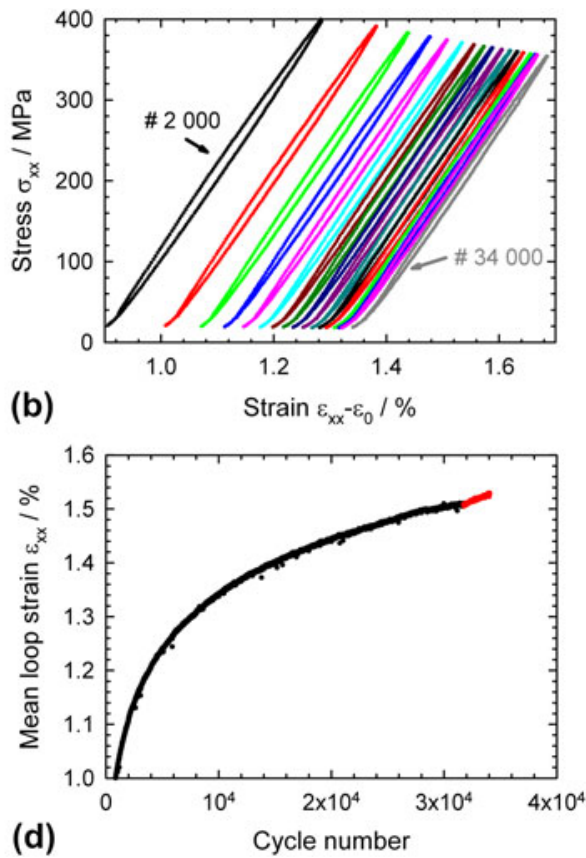

FIG. 6. Quantitative evaluation of the fatigue test of a $230 \mathrm{~nm}$ freestanding gold film at $0.2 \mathrm{~Hz}$ and $50 \mathrm{kPa}$ until failure after $34,058 \mathrm{cycles}$ : (a) planestress modulus evolution, (b) stress-strain loops for every 2000th cycle, (c) corresponding stress and strain ranges, and (d) mean strain $\sigma_{x x}$ during each hysteresis loop. 


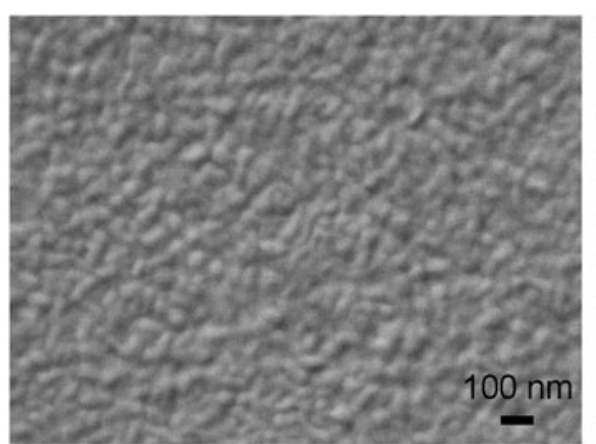

(a)

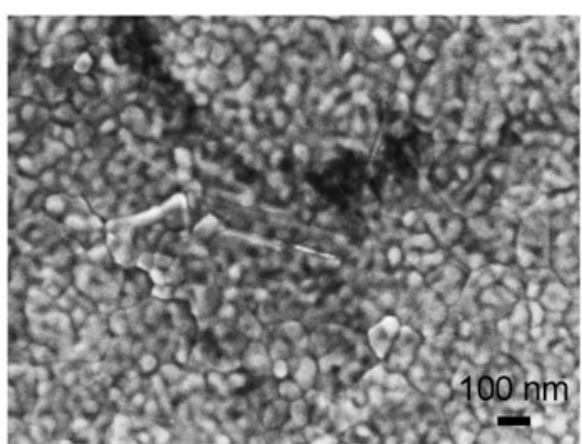

(b)

FIG. 7. SEM images of the surface: (a) before testing and (b) after 25,000 cycles, grains become visible in the topography.

gold membrane tested with the same parameters as used for $\mathrm{SiN}_{x}$ films (a cycling frequency of $0.2 \mathrm{~Hz}$ and a maximum pressure of $50 \mathrm{kPa}$ ). The quantitative evaluation of the test is shown in Fig. 6. This freestanding gold film failed after 34,058 cycles, corresponding to 2 days of testing. Two similar 230-nm-thick films were tested, one of which failed after 13,000 cycles only, while the second one withstood more than 70,000 cycles. It can be seen in Fig. 6(a) that the plane-strain modulus of the film remains fairly constant throughout the test, just as found for $\mathrm{SiN}_{x}$ membranes and gold films on $\mathrm{SiN}_{x}$ substrate. Unlike the previous cases, the evolution of the residual stress is not plotted, because it is nonexistent. The small initial tensile residual stress is indeed already eliminated by the plastic deformation taking place during the first cycle. Being freestanding, the film does not accommodate further plastic deformation by building up a compressive internal stress but would rather buckle if the pressure would be completely removed. The increase of the accumulated plastic strain appears in Fig. 6(b) as the shift to higher strains of the successive hysteresis loops. This shift follows a logarithmic law, which is consistent with cyclic creep being responsible for it. A side effect of this strain increase is that - as the bulge setup is pressure controlledthe stress and strain ranges are slightly reduced, see Fig. 6(c). In Fig. 6(b), a progressive reduction of the hysteresis and the strain increment between two consecutive loops is observed, which is attributed to cyclic hardening. Such a behavior is frequently encountered during the push-pull testing of macroscopic fcc metals samples up to the formation of persistent slip bands, provided the plastic strain range exceeds $\sim 10^{-4,36}$ as it is the case here. On the whole, it can be concluded that the freestanding gold films experience a strong ratcheting behavior. Looking more carefully at Fig. 6(b), one can see that the very last hysteresis loop is slightly out of line compared with the previous ones, with a strain value higher than expected. This final behavior is more clearly seen if the strain data is analyzed from each cycle. Figure 6(d) shows that the plastic strain of the membrane increases at an accelerated rate over the last 2000 cycles preceding failure. This probably corresponds to strain localization leading to the final failure of the sample.

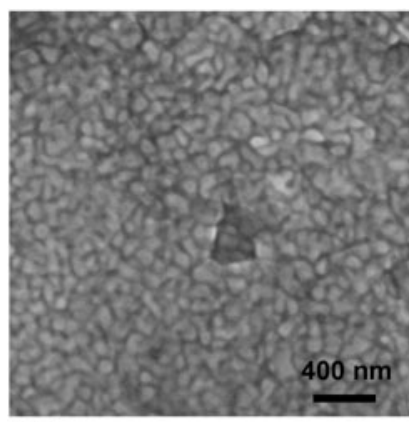

(a)

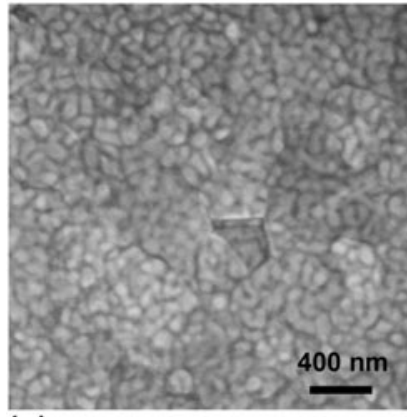

(c)

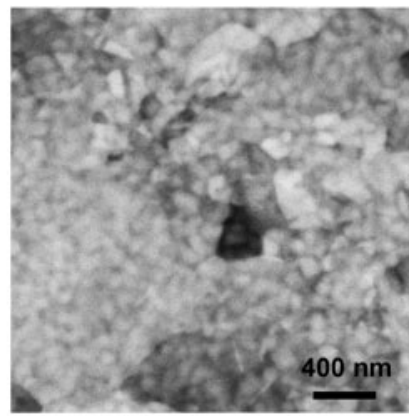

(b)

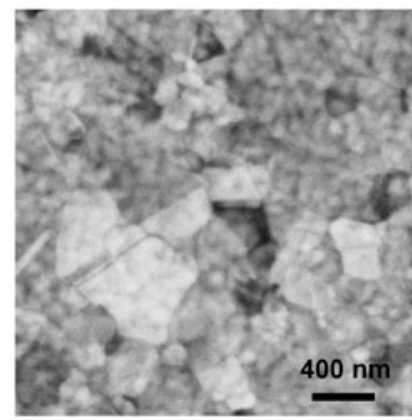

(d)

FIG. 8. SEM images after 70,000 cycles showing sink-in deformation of grains: (a and c) secondary electron contrast and (b and d) backscattered electron contrast.

\section{Microscopic observations}

Several tests on freestanding gold films were interrupted at a periodical interval of 5000 or 10,000 cycles so as to allow inspection of the surface in the optical microscope, SEM, and AFM. The samples had a thickness of about $230 \mathrm{~nm}$ and were tested at a maximum pressure of $30 \mathrm{kPa}$. The first interesting observation is a progressive roughening of the film. While the surface is originally quite smooth, the grain structure becomes visible from the surface topography after a few thousands cycles, see Fig. 7. This roughening of the surface at the grain boundaries is first limited to few areas but becomes more and more widespread as testing goes on. After a longer testing time, it is found that individual grains sink below the original level of the surface 
at various places, see Fig. 8. This sink-in is likely to be a further step of the previously observed surface roughening. The displaced grains have a size of $200-400 \mathrm{~nm}$, therefore neither falling into the categories of the largest nor the smallest grains present in the film. They also have various shapes. It is however striking that they appear rather dark on the micrographs with backscattered electron contrast, which could hint at a common orientation. Investigations by AFM directly confirmed that the contrast that was observed by secondary electron imaging was of topographical nature. Figure 9(a) shows the sink-in of a grain imaged after 70,000 cycles. The AFM cross-profile in Fig. 9(b) reveals that the surface of the displaced grain is tilted away from the horizontal, indicating grain rotation. The sharp vertical line visible on the right side of the diagram strongly suggests grain boundary sliding. A pile-up is also observed within the neighboring grain, presumably as a result of the combined rotation and gliding of the first grain along their common grain boundary. A similar sink-in event of a grain was also investigated with a focused ion beam, see Fig. 10. The crosssection reveals a step extending between both sides of the membrane, which is in line with the assumption of grain boundary sliding associated with limited plasticity. In this particular case, sliding seems to have occurred along a twin boundary. Sliding along other grain boundaries was also found, compare with Fig. 8.

\section{DISCUSSION}

The most striking observation concerns the ratcheting behavior of all fatigue-tested gold films, which is indicative of plastic creep. The magnitudes are however very different for freestanding and $\mathrm{SiN}_{x}$-supported films, with the cyclic creep contribution being roughly $10^{2}$ times higher for the investigated freestanding sample than for the corresponding supported film. The testing parameters used for both films are compared in Table I. It can be seen that they were tested at a similar mean stress level. It should however be noted thatdue to the limited maximum pressure achievable with the experimental setup - a thinner film had to be used on the $\mathrm{SiN}_{x}$ substrate, which implies that its microstructure was finer. ${ }^{29}$ This probably leads to a greater strength of this sample. ${ }^{37,38}$ In any case, the settings used for both kinds of samples correspond to low cycle fatigue testing above their initial yield limit, so the large difference in cyclic creep is not directly connected to this difference of strength, but rather to the different deformation mechanisms that have been observed.

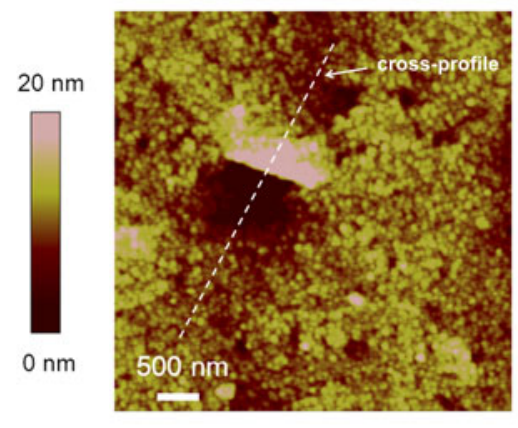

(a)

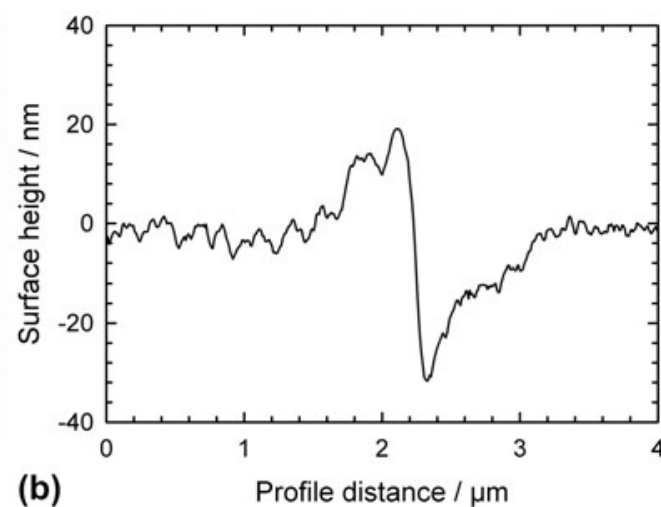

(b)

FIG. 9. AFM micrograph showing the sink-in of a grain after 70,000 cycles (a) and the corresponding cross-profile (b).

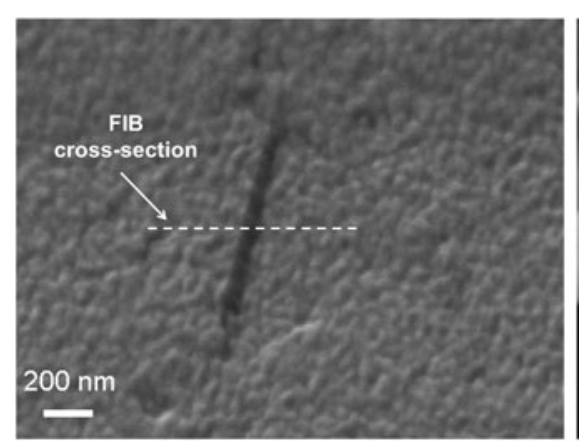

(a)

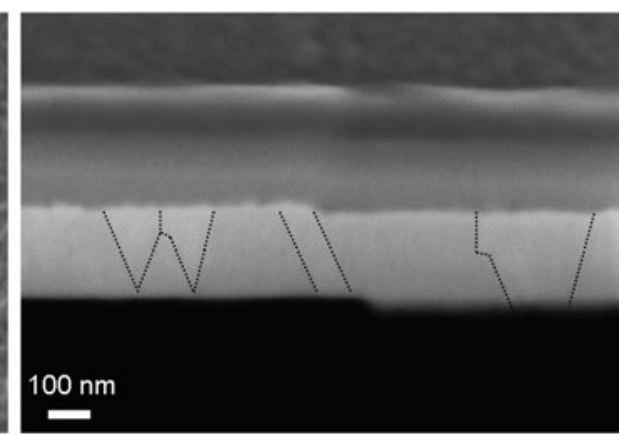

(b)

FIG. 10. Electron microscopic and focused ion beam investigation of grain boundary sliding. (a) Top view of the surface and (b) cross-section showing a step on both sides of the film (grain boundaries visible from secondary electron contrast have been highlighted). 
B. Merle et al.: Bulge fatigue testing of freestanding and supported gold films

TABLE I. Comparison of the main fatigue testing parameters for the freestanding and supported films discussed throughout the article. The values are related to the last cycles of the tests.

\begin{tabular}{lcccccc}
\hline \hline Gold film type & Film thickness (nm) & Mean strain $(\%)$ & Mean stress (MPa) & Strain range (\%) & Stress range (MPa) & Fatigue life \\
\hline Freestanding & 230 & 1.5 & 175 & 0.3 & 330 & $3.4 \times 10^{4}$ cycles \\
Supported by $\mathrm{SiN}_{x}$ & 100 & 0.3 & 190 & 0.6 & 750 & $>10^{5}$ cycles \\
\hline \hline
\end{tabular}

The observations performed after completion of the fatigue tests can be summarized as follows. For the gold films tested on a $\mathrm{SiN}_{x}$ substrate, there is no major change in topography. The most striking modification is a uniform roughening of the surface, which is a consequence of the uniform straining of the upper gold layer by the elastic $\mathrm{SiN}_{x}$ substrate. Its magnitude $\left(0.4 \mathrm{~nm}\right.$ increase of $\left.R_{a}\right)$ however makes it nonhazardous for the fatigue life of the film. Besides this uniform roughening, only few and very localized sites of plastic activity could be detected. On films as thin as $100 \mathrm{~nm}$, the formation of whiskers and hillocks was observed. The likely explanation is that material is squeezed out of the film through weak points in the microstructure when it is put under compression. Only films with a thickness of $300 \mathrm{~nm}$ exhibit the typical extrusions usually associated with the fatigue of coarsegrained bulk samples, and even then they are confined to some abnormally large grains with a diameter of the order of $1 \mu \mathrm{m}$. This is a direct hint that the development of dislocation structures requires a minimum spacing and explains why extrusions were not observed in the 100-nm-thick samples. The good adhesion provided by the chromium interlayer also makes the necessary debonding more difficult, therefore restricting the formation of extrusions. All the findings reported so far are in line with the results obtained by other researchers on thin copper and silver films on polymeric and silicon oxide substrates. ${ }^{1,2,5,9,39,40}$ It should however be mentioned that no cracking at grain boundaries could be observed, even in the thinnest $100 \mathrm{~nm}$ samples. This might be because the strain range was not as high as for the experiments reported in Refs. 9 and 40.

Freestanding films exhibit in comparison a lot of plastic damage. The deformation is mostly localized at grain boundaries and there is repeated evidence of grain boundary sliding. This mechanism is obviously favored by the columnar microstructure of the films, which results in a weak geometrical constraint against the out of plane motion of the grains. Such a motion would be unlikely in nanocrystalline bulk materials because of geometrical incompatibilities with other grains. During nanoindentation experiments, such grain boundary sliding has been exclusively observed at the free surface. ${ }^{41}$ Obviously, it is also unlikely to happen in a film tightly bonded to a substrate. In such a case, and provided that the deformation cannot be carried by intragranular plasticity, one would expect grain boundary cracking. Although the tested freestanding films statistically had just as many abnormally large grains as the films on a $\mathrm{SiN}_{x}$ substrate, not a single extrusion could be found. This is an indication that accommodation of the stress happens more easily by deformation at grain boundaries than by intragranular plasticity.

A first consequence of the observed grain boundary sliding is that it leads to an increase in the sample length. This phenomenon therefore boosts the cyclic creep rate of the freestanding films compared with the supported films, in which grain boundary sliding cannot occur due to the tight bonding to the elastic $\mathrm{SiN}_{x}$ substrate. Another reason for the slower creep rate of the supported films is that, as testing goes on, the gold film becomes increasingly plastically deformed, so a greater part of the load transfers to the substrate. This fast drop of the mean stress is clearly visible in Fig. 3(b). It reduces the driving force for creep in the gold film and is not compensated in any way by the substrate, which is elastic and does not creep. The effect would be less pronounced for a more compliant substrate. Another consequence of the localized deformation at grain boundaries is the associated strong roughening of the surface, which is likely to cause strain localization leading to an early failure of the film. This might account for the poorer fatigue life of the freestanding sample, although it was tested at stress and strain ranges half as large only as for the $\mathrm{SiN}_{x}$ supported film (see Table I). A comprehensive study of the fatigue life of the films would require constructing S-N curves, which did not prove so far possible due to the limited number of samples available. From the literature, ${ }^{5}$ it is expected that supported films follow the Coffin-Manson law, given a proper failure criterion is adopted. ${ }^{9,10}$ The bulge fatigue technique could be conveniently used in the future to test this assumption on freestanding films.

\section{CONCLUSION}

In this study, the bulge test was used to study the low cycle fatigue properties of thin films up to $\sim 10^{5}$ cycles. The excellent stability of the setup was demonstrated by tests on the reference material LPCVD $\mathrm{SiN}_{x}$, which is known to be insensitive to fatigue at the considered stress level. The fatigue damage mechanisms found on gold films on $\mathrm{SiN}_{x}$ substrate are consistent with previous observations reported in the literature. More interesting are the findings on freestanding gold films of about $200 \mathrm{~nm}$ thickness, for which there are hardly any experimental data available so far. There, the deformation occurs almost 
exclusively at or around grain boundaries, with numerous occurrences of grain boundary sliding. This sliding contributes to the elongation of the sample and therefore might at least partially account for the much higher cyclic creep rate measured compared to gold films on $\mathrm{SiN}_{x}$ substrate tested at the same stress level. This phenomenon occurs presumably because the microstructure of the films is mostly columnar; therefore, there is no strong geometrical constraint acting against the out of plane motion of the grains. Grain boundary sliding also results in the local thinning of the film, which might prompt strain localization and reduce the fatigue life of the sample.

\section{ACKNOWLEDGMENTS}

The authors would like to acknowledge the kind support received from Petra Rosner from the group of Erdmann Spiecker in Erlangen for the deposition of the gold films. They also thank Heinz Werner Höppel for his valuable advice about the fatigue tests, as well as Haël Mughrabi for useful discussions about the manuscript. The authors also gratefully acknowledge the funding of the German Research Council (DFG), which, within the framework of its "Excellence Initiative," supports the cluster of excellence "Engineering of Advanced Materials" at the University of Erlangen-Nürnberg.

\section{REFERENCES}

1. R. Schwaiger, G. Dehm, and O. Kraft: Cyclic deformation of polycrystalline Cu films. Philos. Mag. 83(6), 693-710 (2003).

2. R. Schwaiger and O. Kraft: Size effects in the fatigue behavior of thin Ag films. Acta Mater. 51(1), 195-206 (2003).

3. G.P. Zhang, C.A. Volkert, R. Schwaiger, P. Wellner, E. Arzt, and O. Kraft: Length-scale-controlled fatigue mechanisms in thin copper films. Acta Mater. 54(11), 3127-3139 (2006).

4. N. Lu, Z. Suo, and J.J. Vlassak: The effect of film thickness on the failure strain of polymer-supported metal films. Acta Mater. 58(5), 1679-1687 (2010).

5. O. Kraft, R. Schwaiger, and P. Wellner: Fatigue in thin films: Lifetime and damage formation. Mater. Sci. Eng., A 319-321, 919-923 (2001).

6. H. Mughrabi: Dislocation wall and cell structures and long-range internal stresses in deformed metal crystals. Acta Metall. 31(9), 1367-1379 (1983).

7. H. Mughrabi: Cyclic slip irreversibilities and the evolution of fatigue damage. Metall. Mater. Trans. B 40(4), 431-453 (2009).

8. A.T. Winter: Etching studies of dislocation microstructures in crystals of copper fatigued at low constant plastic strain amplitude. Philos. Mag. 28(1), 57-64 (1973).

9. G-D. Sim, Y. Hwangbo, H-H. Kim, S-B. Lee, and J.J. Vlassak: Fatigue of polymer-supported Ag thin films. Scr. Mater. 66(11), 915-918 (2012).

10. X.J. Sun, C.C. Wang, J. Zhang, G. Liu, G.J. Zhang, X.D. Ding, G.P. Zhang, and J. Sun: Thickness dependent fatigue life at microcrack nucleation for metal thin films on flexible substrates. J. Phys. D: Appl. Phys. 41(19), 195404 (2008).

11. N. Lu, X. Wang, Z. Suo, and J. Vlassak: Failure by simultaneous grain growth, strain localization, and interface debonding in metal films on polymer substrates. J. Mater. Res. 24(2), 379-385 (2009).
12. K. Abbas, Z.C. Leseman, and T.J. Mackin: Ultra low cycle fatigue of axisymmetric freestanding nanoscale gold films. In ASME Proceedings, ASME: 2008; pp. 91-97.

13. M-T. Lin, C-J. Tong, and KS. Shiu: Novel microtensile method for monotonic and cyclic testing of freestanding copper thin films. Exp. Mech. 50(1), 55-64 (2010).

14. M-T. Lin, C-J. Tong, and K-S. Shiu: Monotonic and fatigue testing of freestanding submicron thin beams application for MEMS. Microsys. Technol. 14(7), 1041-1048 (2008).

15. O. Kraft and C.A. Volkert: Mechanical testing of thin films and small structures. Adv. Eng. Mater. 3(3), 99-110 (2001).

16. E.W. Schweitzer and M. Göken: In situ bulge testing in an atomic force microscope: Microdeformation experiments of thin film membranes. J. Mater. Res. 22(10), 2902-2911 (2007).

17. B. Merle and M. Göken: Fracture toughness of silicon nitride thin films of different thicknesses as measured by bulge tests. Acta Mater. 59(4), 1772-1779 (2011).

18. J.A. Liddle, H.A. Huggins, P. Mulgrew, L.R. Harriott, H.H. Wade, and K. Bolan: Fracture strength of thin ceramic membranes. Mater. Res. Soc. Symp. Proc. 338, 501-506 (1994).

19. Y. Xiang, J. McKinnell, W-M. Ang, and J.J. Vlassak: Measuring the fracture toughness of ultra-thin films with application to alta coatings. Int. J. Fract. 144(3), 173-179 (2007).

20. A.J. Kalkman, A.H. Verbruggen, and G.C.A.M. Janssen: Young's modulus measurements and grain boundary sliding in free-standing thin metal films. Appl. Phys. Lett. 78(18), 2673-2675 (2001).

21. J.J. Vlassak and W.D. Nix: New bulge test technique for the determination of Young's modulus and Poisson's ratio of thin films. J. Mater. Res. 7(12), 3242-3249 (1992).

22. Y. Xiang, X. Chen, and J.J. Vlassak: Plane-strain bulge test for thin films. J. Mater. Res. 20(9), 2360-2370 (2005).

23. M. Small and W.D. Nix: Analysis of the accuracy of the bulge test in determining the mechanical properties of thin films. J. Mater. Res. 7(6), 1553-1563 (1992).

24. U.F. Kocks, C.N. Tomé, and H-R. Wenk: Texture and Anisotropy (Cambridge University Press, Cambridge, UK, 1998).

25. B.A. Movchan and A.V. Demchishin: Study of the structure and properties of thick vacuum condensates of nickel, titanium, tungsten, aluminum oxide and zirconium dioxide. Fiz. Met. Metalloved. 28(4), 653-660 (1969).

26. W-H. Chuang, R.K. Fettig, and R. Ghodssi: Nano-scale fatigue study of LPCVD silicon nitride thin films using a mechanicalamplifier actuator. J. Micromech. Microeng. 17(5), 938-944 (2007).

27. W-H. Chuang, R.K. Fettig, and R. Ghodssi: Fatigue study of nanoscale silicon nitride thin films using a novel electrostatic actuator. In Digest of Technical Papers Transducers '05, IEEE: 2005; pp. 1957-1960.

28. D. Callister, Jr.: Fundamental of Material Science and Engineering (Wiley \& Sons, New York, 2005).

29. B. Merle, E.W. Schweitzer, and M. Göken: Thickness and grain size dependence of the strength of copper thin films as investigated with bulge tests and nanoindentations. Philos. Mag. 92(25-27), 3172-3187 (2012).

30. G. Simmons and H. Wang: Single Crystal Elastic Constants and Calculated Aggregate Properties: A Handbook (The MIT Press, Cambridge, MA, 1971)

31. Y. Xiang and J.J. Vlassak: Bauschinger effect in thin metal films. Scr. Mater. 53(2), 177-182 (2005).

32. M. Hommel, O. Kraft, and E. Arzt: A new method to study cyclic deformation of thin films in tension and compression. J. Mater. Res. 14(6), 2373-2376 (1999).

33. C. Eberl, R. Spolenak, E. Arzt, F. Kubat, A. Leidl, W. Ruile, and O. Kraft: Ultra high-cycle fatigue in pure Al thin films and line structures. Mater. Sci. Eng., A 421(1-2), 68-76 (2006). 
34. I.A. Blech: Electromigration in thin aluminum films on titanium nitride. J. Appl. Phys. 47(4), 1203-1208 (1976).

35. D-K. Kim, B. Heiland, W.D. Nix, E. Arzt, M.D. Deal, and J.D. Plummer: Microstructure of thermal hillocks on blanket $\mathrm{Al}$ thin films. Thin Solid Films 371(1), 278-282 (2000).

36. H. Mughrabi, F. Ackermann, and K. Herz: Persistent slip bands in fatigued face-centered and body-centered cubic metals, ASTM Special Technical Publication 675, 69-105 (1979).

37. E. Arzt: Size effects in materials due to microstructural and dimensional constraints: A comparative review. Acta Mater. 46(16), 5611-5626 (1998).
38. W.D. Nix: Mechanical properties of thin films. Metall. Trans. A 20(11), 2217-2245 (1989).

39. R. Schwaiger and O. Kraft: High cycle fatigue of thin silver films investigated by dynamic microbeam deflection. Scr. Mater. 41(8), 823-829 (1999).

40. D. Wang, C.A. Volkert, and O. Kraft: Effect of length scale on fatigue life and damage formation in thin $\mathrm{Cu}$ films. Mater. Sci. Eng. A 493(1-2), 267-273 (2008).

41. V. Maier, B. Merle, M. Göken, and K. Durst: An improved longterm nanoindentation creep testing approach for studying the local deformation processes in nanocrystalline metals at room and elevated temperatures. J. Mater. Res. 28(9), 1177-1188 (2013). 